\title{
Loss of PTEN is associated with elevated EGFR and HER2 expression and worse prognosis in salivary gland cancer
}

\author{
T Ettl', , K Baader', C Stiegler², M Müller ${ }^{3}$, A Agaimy ${ }^{3}$, J Zenk ${ }^{4}$, T Kühnel' ${ }^{5}$, M Gosau', K Zeitler², \\ S Schwarz ${ }^{3,7}$ and G Brockhoff ${ }^{6,7}$
}

'Department of Oral and Maxillofacial Surgery, University of Regensburg, Franz-Josef-Strauss-Allee II, 93053 Regensburg, Germany; ${ }^{2}$ Department of Pathology, University of Regensburg, Franz-Josef-Strauss-Allee II, 93053 Regensburg, Germany; ${ }^{3}$ Department of Pathology, University of ErlangenNuremberg, Krankenhausstraße 12, 91054 Erlangen, Germany; ${ }^{4}$ Department of Otorhinolaryngology, University of Erlangen-Nuremberg, Waldstraße I, 91054 Erlangen, Germany; ${ }^{5}$ Department of Otorhinolaryngology, University of Regensburg, Franz-Josef-Strauss-Allee II, 93053 Regensburg, Germany; ${ }^{6}$ Department of Gynecology and Obstetrics, University of Regensburg, Landshuter Straße 65, 93053 Regensburg, Germany

\begin{abstract}
BACKGROUND: Activity of the tumour-suppressor gene PTEN is reduced in different types of cancer and implicates non-responsiveness to targeted therapy. This study evaluates the gene and protein status of PTEN in salivary gland carcinomas.

METHODS: A total of 287 carcinomas of the major and minor salivary glands were investigated for phosphatase and tensin homologue located on chromosome 10 (PTEN) deletion and loss of PTEN expression using fluorescence in situ hybridisation (FISH) and immunohistochemistry $(\mathrm{IHC})$, respectively. Results were correlated to clinicopathological parameters, long-term survival, epidermal growth factor receptor (EGFR) and human epidermal growth factor receptor 2 (HER2) (IHC and FISH) status of the tumours. RESULTS: Hemizygous deletions of PTEN were found in 35 out of 232 (I5.1\%) carcinomas, while homozygous deletions were observed in 17 out of 232 (7.3\%) tumours. Phosphatase and tensin homologue located on chromosome 10 deletion was common in certain histological subtypes and especially homozygous deletion was associated with high-grade malignancy, lymph node metastases and unfavourable long-term prognosis $(P<0.001)$. Loss of PTEN expression was present in 59 out of $273(21.6 \%)$ carcinomas and was significantly correlated to genomic PTEN deletion, high-grade malignancy $(P<0.00 \mathrm{I})$, increased tumour size $(P=0.036)$, lymph node metastases $(P=0.007)$ and worse disease-specific survival $(P=0.002)$. Genomic PTEN deletion, in particular homogenous deletion $(P<0.001)$ predominantly occurred in tumours with increased gene copy number of EGFR $(60.0 \%)$ and/or amplification of HER2 (63.6\%). Loss of PTEN expression was frequently found in tumours overexpressing EGFR (28.6\%) and/or HER2 (52.6\%). CONCLUSION: PTEN function is reduced in different types of salivary gland cancer indicating unfavourable prognosis. Its association with EGFR and HER2 signalling might affect targeted therapy.

British Journal of Cancer (2012) I 06, 719-726. doi:I0.1038/bjc.2011.605 www.bjcancer.com

Published online 12 January 2012

(C) 2012 Cancer Research UK
\end{abstract}

Keywords: salivary gland cancer; PTEN; deletion; prognosis; EGFR HER2

Salivary gland carcinomas are rare tumours with an annual incidence rate between 0.4 and 2.6 per 100 (Eveson et al, 2005). As the current World Health Organisation (WHO) classifies 24 different malignant subtypes with varying clinical courses and prognoses, diagnosis and treatment of these tumours represent a special challenge for both pathologists and surgeons. Recent attempts of treatment for unresectable or metastatic disease comprise targeted therapies against the epidermal growth factor receptor (EGFR, ErbB1) and the human epidermal growth factor receptor 2 (HER2, ErbB2) (Haddad et al, 2003; Agulnik et al, 2007; Locati et al, 2008). To date, however, response rates are rather disappointing.

Phosphatase and tensin homologue located on chromosome 10 (PTEN) is a tumour-suppressor gene frequently lost on chromosome 10q23 in different types of cancer (Li et al, 1997).

\footnotetext{
*Correspondence: Dr T Ettl; E-mail: tobias.ett|@klinik.uni-regensburg.de

${ }^{7}$ These authors equally contributed to the results of this work.

Received 5 July 20 I I; revised 2 December 201 I; accepted 21 December 201 I; published online 12 January 2012
}

In the nucleus, PTEN regulates genomic stability, cell cycle progression, differentiation and gene expression (Carracedo et al, 2011). Beside these tumour-suppressive activities, PTEN functions as a negative regulator of the PI3K pathway by dephosphorylating the $3^{\prime}$ position of phosphoinositide 3,4,5-triphosphate (PIP3). Stimulated by EGFR or HER2, PI3K activates the Ser/Thr kinase AKT, thus promoting cell survival and tumour growth (Courtney et al, 2010). Hemi- or homozygous deletions of PTEN are frequent events in prostate cancer and highgrade gliomas with unfavourable impact on prognosis (Korshunov et al, 2004; Yoshimoto et al, 2007). Loss of PTEN seems associated with unresponsiveness to targeted therapy against EGFR in colorectal cancer and glioblastomas (Mellinghoff et al, 2005; Frattini et al, 2007; Negri et al, 2010), moreover it predicts resistance to trastuzumab in HER2-positive breast cancer (Nagata et al, 2004; Pandolfi, 2004). The purpose of this study was to evaluate the gene and protein status of PTEN in a representative cohort of salivary gland carcinomas and to correlate the results to clinicopathological parameters, to long-term survival, and to the gene and protein status of EGFR and HER2. 


\section{PATIENTS AND METHODS}

\section{Patients and treatment modalities}

The study comprised 287 patients with carcinomas of the major and minor salivary glands, diagnosed at the Departments of Pathology of the University Hospital Regensburg, the University Hospital Erlangen-Nuremberg and the Hospital Clinic Nuremberg between 1984 and 2008. Clinical and follow-up data were obtained from the clinical tumour registries of Regensburg and ErlangenNuremberg in accordance with the Research Ethic Guidelines of the medical faculties. At diagnosis, patients were staged according to the TNM system of the Union for International Cancer Control (UICC) (Sobin et al, 2009). Tumour surgery was performed at the Departments of Oral and Maxillofacial Surgery and Otorhinolaryngology of Regensburg University Hospital, Erlangen-Nuremberg University Hospital and Nuremberg City Hospital. A total of 287 patients underwent primary surgery; lymph node dissection was performed for $205(71.9 \%)$ patients. Postoperative radio- or radiochemotherapy was applied in $170(59.2 \%)$ cases with highgrade malignancy, positive resection margins, lymph node metastases or distant metastasis.

A total of $204(71.1 \%)$ parotid, 39 (13.6\%) submandibular, $2(0.7 \%)$ sublingual and $42(14.6 \%)$ minor gland carcinomas were recorded. In all, $56.1 \%$ of the patients presented with advanced (III, IV) UICC tumour stages at diagnosis. Cervical lymph node metastases were obvious in $31.8 \%$, distant metastases occurred in $10.6 \%$ of the patients. In 217 cases $(77.8 \%)$, close resection margins (R0) were achieved, 48 (17.2\%) patients ended up with microscopic (R1) and $14(5.0 \%)$ patients with macroscopic (R2) residual tumour after surgery. With view to the tumours originating from the minor salivary glands clear margins were achieved in $73.8 \%$ (31 out of 42 ). Ten $(23.8 \%)$ carcinomas presented microscopic residual tumour and one minor gland carcinoma $(2.4 \%)$ ended up with macroscopic residual tumour after surgery.

\section{Follow-up studies}

In total, 137 male and 150 female patients showed a mean age of $60.6(11-99)$ years at diagnosis. The mean follow-up of all patients was 4.75 (range $0.1-21.2$ ) years. Recurrence was observed in $74(26.8 \%)$ patients. Disease-related deaths occurred in $73(25.4 \%)$ cases. The 5- and 10-year disease-specific survival rates of all patients were $73.2 \%$ and $68.1 \%$, respectively.

\section{Histology and classification}

Haematoxylin-eosin slides from paraffin wax-embedded tumours were available for all cases and were independently reviewed by two experienced pathologists (SS and AA) without knowledge of the initial diagnosis. All tumours were classified according the contemporary WHO's classification of salivary gland tumours (Barnes et al, 2005). The study cohort comprised 40 acinic cell carcinomas (ACCCs), 52 adenoid cystic carcinomas (ACCs), 45 mucoepidermoid carcinomas (MECs), 27 salivary duct carcinomas (SDCs), 31 adenocarcinomas NOS (ACNOS), 28 squamous cell carcinomas (SQCCs), 21 myoepithelial carcinomas (MYECs), 10 polymorphous low-grade adenocarcinomas (PLGAs), 9 basal cell adenocarcinomas (BCACs), 9 oncocytic carcinomas (OCCs), 4 epithelial-myoepithelial carcinomas (EMCs), 4 malignant mixed tumours (MMTs), 4 undifferentiated carcinomas (UCs), 2 large cell carcinomas (LCCs) and 1 cystadenocarcinoma (CACs). The less frequent entities PLGA, BCAC, OCC, EMC, MMT, UC, LCC and CAC were summarised as 'others' in Table 1. All cases of SQCC were classified as primitive of the salivary glands after intensive staging procedures (CT or MRI of the head and neck, panendoscopy, X-ray or CT of the chest and ultrasonograpy of the abdomen) and exclusion of a metastasis to the salivary gland.
Squamoid variants of MECs were thoroughly sorted out (Schwarz et al, 2011). Grading was based on a three-tiered grading system (Therkildsen et al, 1998; Jouzdani et al, 2010). The ACCC, BCAC, EMC, CAC and PLGA were considered low grade (G1) with the exception of dedifferentiated tumours, which were classified high grade (G3). In contrast, SDC, SQCC, MMT, OCC, UC and LCC were classified high grade (G3). Mucoepidermoid carcinomas were graded according to the criteria proposed in the current WHO classification (Barnes et al, 2005). Adenoid cystic carcinomas were divided into predominantly tubulo-cribriform (G2) and predominantly solid (G3) tumours. Grading of ACNOS and MYEC was based on nuclear pleomorphism and mitotic activity similar to the Elston and Ellis grading of breast cancer (Elston and Ellis, 1991). The 27 cases of carcinoma ex pleomorphic adenoma were classified and graded according to the malignant component of the tumour.

\section{Immunohistochemistry}

A tissue microarray (TMA) with $2.0 \mathrm{~mm}$ diameter punch cores was constructed from formalin-fixed paraffin-embedded tissue blocks of all patients as previously described (Milanes-Yearsley et al, 2002). Haematoxylin-eosin-stained TMA sections were used for reference histology.

Immunostaining of PTEN (Cell Signaling Technology, Inc., Danvers, MA, USA, 138G6, \#9559, monoclonal rabbit, dilution 1:50, detection EnVision Dual Link System, Dako, Glostrup, Denmark), EGFR (Dako, EGFR pharmDx, clone 2-18C9, monoclonal mouse, dilution 1:400, detection En Vision, Dako) and HER2 (Dako, A0485, polyclonal rabbit, dilution $1: 250$, detection iVIEW DAB, Ventana Medical Systems, Inc., Illkirch, France) was performed on $5 \mu \mathrm{m}$ sections of the TMAs and applied according to the manufacturer's instructions. After dewaxing, washing and rehydration of the slides through xylene and graded alcohols, microwave heating in citrate buffer was used for antigen retrieval. In case of EGFR, proteinase $\mathrm{K}$ was applied for epitope retrieval. Endogenous peroxidase was blocked in ChemMate peroxidaseblocking solution (Dako). Immunohistochemistry (IHC) for PTEN was semiquantitatively evaluated based on nuclear and cytoplasmic staining (Figure 1A-C). An immunoreactive score (IRS) was built as the product of staining intensity (none $=0$, weak $=1$, moderate $=2$, strong $=3$ ) and the percentage of positive tumour cells $(0-100 \%)$ resulting in an IRS ranging from 0 to 300 points (Laurent-Puig et al, 2009). Tumours were dichotomised into PTEN-negative (IRS 0-59) and PTEN-positive (IRS 60-300). The immunostaining of EGFR and HER2 was semiquantitatively evaluated based on intensity of membrane reactivity following the original DAKO Herceptest criteria with a threshold of $10 \%$ immunopositive cells: 0 , negative (no reactivity or reactivity in $<10$ of cells); $1+$, weak reactivity in $>10 \%$ of cells, $2+$ moderate reactivity in $>10 \%$ cells; $3+$ strong reactivity in $>10 \%$ cells. Tumours with $3+$ staining were considered as positive (Figure 1G and $\mathrm{H}$ ). Immunostaining pattern was also documented in normal salivary gland tissues as controls.

\section{Fluorescence in situ hybridisation}

As described in detail elsewhere (Sassen et al, 2008) TMA sections were mounted on charged slides (SuperFrost Plus; Menzel GmbH, Braunschweig, Germany). Haematoxylin-eosin-stained TMA sections were used for reference histology. Fluorescence in situ hybridisation (FISH) was performed with the use of directly labelled ZytoLight SPEC PTEN/CEN10, SPEC EGFR/CEN7 and SPEC HER2/CEN17 dual colour probes (ZytoVision Ltd, Bremerhaven, Germany). After probe, hybridisation nuclei were counterstained with anti-fading 4',6-diamidino-2-phenylindole Vectashield (Vector Laboratories, Burlingame, CA, USA) and were analysed by epifluorescence microscopy using the AxioImagerZ1 (Zeiss, Göttingen, Germany). Hybridisation signals of 
Table I PTEN and clinicopathological parameters

\begin{tabular}{|c|c|c|c|c|c|}
\hline \multirow[b]{2}{*}{ Parameter } & \multicolumn{3}{|c|}{ PTEN FISH } & \multicolumn{2}{|c|}{ PTEN IHC } \\
\hline & Non-deleted & Hemizygous & Homozygous & Pos & Neg \\
\hline ACCC & $30(85.7 \%)$ & $5(14.3 \%)$ & 0 & $36(90.0 \%)$ & $4(10.0 \%)$ \\
\hline ADCC & 41 (97.6\%) & I (2.4\%) & 0 & 42 (84.0\%) & $8(16.0 \%)$ \\
\hline MEC & 35 (89.7\%) & $3(7.7 \%)$ & | (2.6\%) & $36(85.7 \%)$ & $6(14.3 \%)$ \\
\hline SDC & 8 (33.3\%) & $9(37.5 \%)$ & 7 (29.2\%) & $14(58.3 \%)$ & $10(41.7 \%)$ \\
\hline MYEC & $15(83.3 \%)$ & $2(11.1 \%)$ & I (5.6\%) & $19(95.0 \%)$ & | (5.0\%) \\
\hline Others & 25 (13.9\%) & I (2.7\%) & 0 & $31(72.1 \%)$ & $12(27.9 \%)$ \\
\hline \multicolumn{6}{|l|}{ Age } \\
\hline$<70$ & $108(82.4 \%)$ & 15 (1 1.5\%) & $8(6.1 \%)$ & | 40 (84.3\%) & $26(15.7 \%)$ \\
\hline$>70$ & $48(68.6 \%)$ & $14(20.0 \%)$ & $8(11.4 \%)$ & 74 (69.2\%) & $33(30.8 \%) * *$ \\
\hline \multicolumn{6}{|l|}{ Grade } \\
\hline \multicolumn{6}{|l|}{ T-stage } \\
\hline $\mathrm{T} 1-\mathrm{T} 2$ & 103 (79.2\%) & $19(14.6 \%)$ & $8(6.2 \%)$ & $130(82.8 \%)$ & $27(17.2 \%)$ \\
\hline T3-T4 & $73(74.5 \%)$ & $16(16.3 \%)$ & $9(9.2 \%)$ & $80(71.4 \%)$ & $32(28.6 \%) *$ \\
\hline \multicolumn{6}{|l|}{ N-stage } \\
\hline No & $134(85.4 \%)$ & $16(10.2 \%)$ & 7 (4.5\%) & $152(82.6 \%)$ & $32(17.4 \%)$ \\
\hline $\mathrm{NI}-3$ & 39 (57.4\%) & $19(27.9 \%)$ & $10(14.7 \%)^{* * * *}$ & $55(67.1 \%)$ & $27(32.9 \%) * *$ \\
\hline \multicolumn{6}{|l|}{ EGFR FISH } \\
\hline No CNG & |3| (82.9\%) & 21 (13.3\%) & $6(3.8 \%)$ & 153 (83.6\%) & $30(16.4 \%)$ \\
\hline CNG & $12(40.0 \%)$ & $8(26.7 \%)$ & $10(33.3 \%)^{* * * *}$ & $18(54.5 \%)$ & I $5(45.5 \%)^{* * * *}$ \\
\hline \multicolumn{6}{|l|}{ EGFR IHC } \\
\hline $0+, 1+, 2+$ & I 23 (80.4\%) & $20(13.1 \%)$ & $10(6.5 \%)$ & $153(81.4 \%)$ & 35 (I8.6\%) \\
\hline \multicolumn{6}{|l|}{ HER2 $I H C$} \\
\hline
\end{tabular}

Abbreviations: $A C C$ = adenoid cystic carcinoma; $A C N O S=$ adenocarcinoma NOS; CNG = copy number gain; $E G F R=$ epidermal growth factor receptor; FISH = fluorescence in situ hybridisation; HER2 = human epidermal growth factor receptor 2; $I \mathrm{HC}=$ immunohistochemistry; MEC = mucoepidermoid carcinoma; MYEC = myoepithelial carcinoma; $\mathrm{Neg}=$ negative; Pos = positive; PTEN = phosphatase and tensin homologue located on chromosome I0; SDC = salivary duct carcinoma; SQCC = squamous cell carcinoma. $* P \leqslant 0.05, * * P \leqslant 0.01, * * * * \leqslant 0.001$. Statistically significant associations are highlighted in bold.

50 non-overlapped nuclei were manually counted on single cell basis by two independent observers. Non-neoplastic salivary gland specimens were used as controls.

Homozygous deletion of PTEN was defined by the simultaneous lack of both PTEN locus signals and by the presence of centromere signals in $>20 \%$ of nuclei. Hemizygous deletion of PTEN was defined as $>30 \%$ of tumour nuclei containing either one PTEN locus signal and $\geqslant 2$ centromere signals or 2 PTEN locus signals and $\geqslant 4$ centromere signals (relative deletions) (Sircar et al, 2009). Representative examples are shown in Figures 1D-F (centromere signals in red).

For EGFR, samples were grouped as normal disomy, $\leqslant 2$ centromere signals in $\geqslant 50 \%$ of cells; low polysomy/trisomy, $\geqslant 3$ centromere signals in $\geqslant 40 \%$ of cells, excluding cases with high polysomy or gene amplification; high polysomy, $\geqslant 4$ centromere signals in $\geqslant 40 \%$ of cells, excluding cases with gene amplification; and gene amplification, ratio of gene/chromosome $\geqslant 2$ or clusters of probes ( $>10$ copies per tumour cell) in $\geqslant 40 \%$ of cells. Disomy and trisomy/low polysomy were grouped as FISH negative, while high polysomy and amplification (Figure 1J) were classified as
FISH positive or copy number gain (CNG) of EGFR in dichotomisation (Chung et al, 2006; Pectasides et al, 2011).

Human epidermal growth factor receptor 2 was evaluated referring to the guideline recommendations for HER2 testing in breast cancer (Wolff et al, 2007). Gene amplification was assessed by a FISH ratio (HER2 gene signals to chromosome 17 signals) of $>2.2$ (Figure 1I).

\section{Statistical analysis}

Data were analysed with SPSS for Windows, version 15.0 (SPSS, Erkrath, Germany). Relationships between parameters were examined using Pearson's $\chi^{2}$-test $(P<0.05)$ and Fisher's exact probability test $(P<0.05)$ for dichotomised variables. Univariate survival curves were calculated by the Kaplan-Meier method and distributions were compared using the log-rank test. Diseasespecific overall survival was calculated from the date of diagnosis until disease-caused death or end of follow-up. Cox proportional hazards model (enter method) was used in multivariate analyses. 

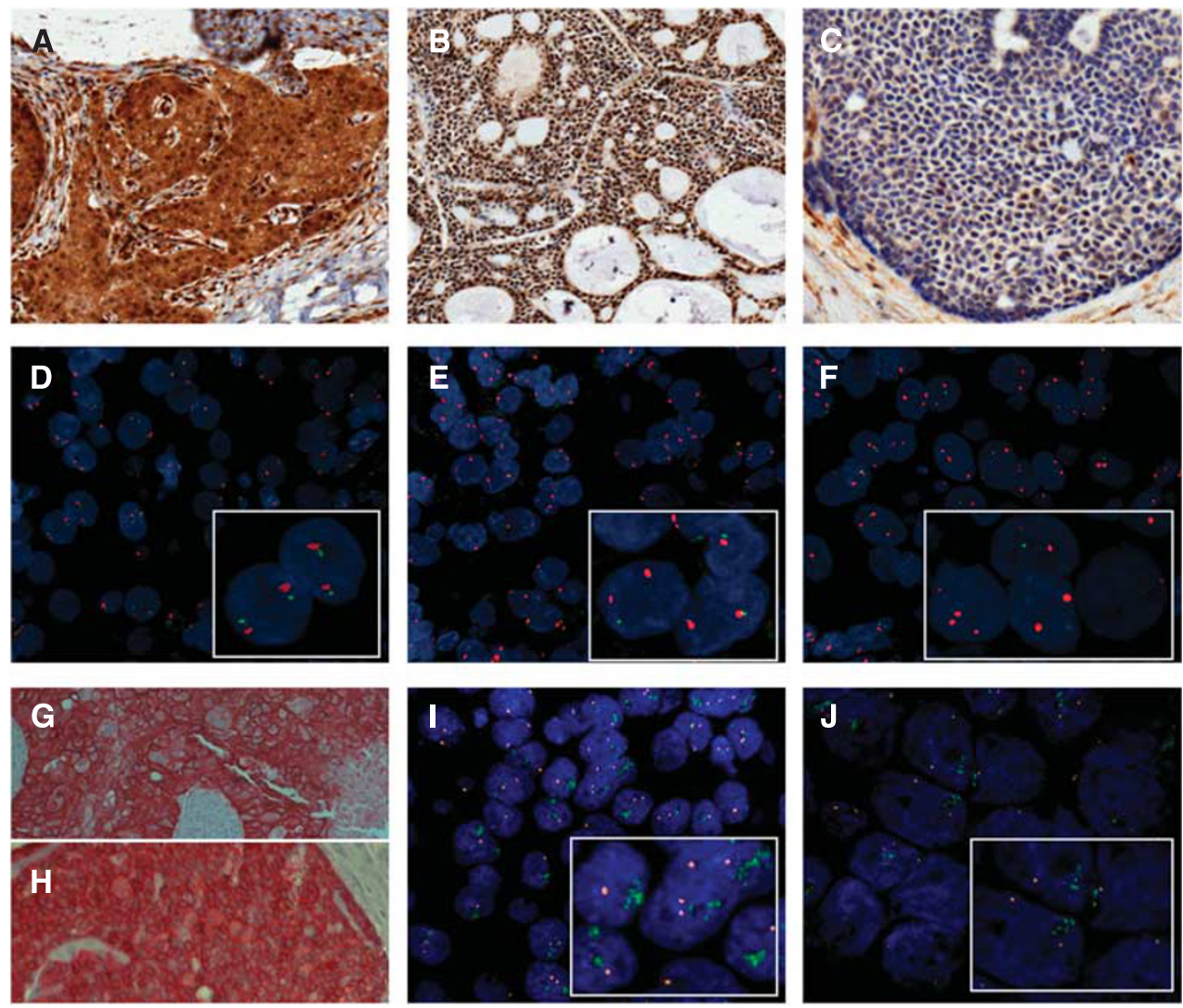

Figure I (A) Strong nuclear and cytoplasmic PTEN immunostaining in ACCC (IRS 300, × 200). (B) Strong nuclear PTEN staining in ACC (IRS 300 $\times$ 100), (C) Weak nuclear PTEN staining in ACC (IRS 10, $\times 200$ ). (D) Disomy of genomic PTEN in ACCC. (E) Hemizygous deletion of PTEN in ACCC. (F) Homozygous deletion of PTEN in SDC. (G) $3+$ staining of HER2 in SDC $(\times 200)$. (H) $3+$ staining of EGFR in ACC $(\times 200)$. (I) Cluster amplification of HER2 in SDC. (J) Cluster amplification of EGFR in MEC.

\section{RESULTS}

\section{PTEN FISH analysis}

A total of 232 salivary gland carcinomas were available for PTEN FISH analysis (Table 1). Hemizygous deletion was found in 35 (15.1\%) tumour samples and homozygous deletion was identified in $17(7.3 \%)$ carcinomas resulting in an overall deletion rate of $22.4 \%(n=52)$. Representative images are shown in Figure 1. Hemi- and homozygous deletions of PTEN were commonly found in SDCs $(66.6 \%)$, ACNOS (50\%) and SQCCs $(42.3 \%)$. One out of three SDCs presented a homozygous deletion. In contrast, deletions were rare events in ACC and MEC (Table 1). Genomic loss of PTEN (hemi- and homozygous deletion) was associated with high-grade malignancy $(P<0.001)$, lymph node metastases $(P<0.001)$ and advanced (III, IV) tumour stage $(P=0.002)$ Homozygous PTEN deletion displayed more aggressive behaviour as $94.1 \%$ (16 out of 17) of these tumours were high-grade carcinomas in contrast to $60.0 \%$ (21 out of 35 ) high-grade malignancies among the tumours with hemizygous deletion of PTEN.

In all, $60.0 \%$ (18 out of 30 ) of the tumours with an increased EGFR copy number presented hemi- or homozygous deletion of PTEN (87.5\% of the SDCs) in contrast to only $17.1 \%$ (27 out of 158) PTEN deletion in EGFR-negative tumours $(P<0.001)$. Phosphatase and tensin homologue located on chromosome 10 deletion was also associated with EGFR protein overexpression (15 out of $42,35.7 \%$ vs 30 out of $153,19.6 \%$ of EGFR-negative tumours, $P=0.038$ ). In total, $63.6 \%$ (7 out of 11 ) of tumours with an amplification of HER2 presented a deletion (6 homozygous) of
PTEN (83.3\% of the SDCs) in comparison with only $20 \%$ (37 out of 185) PTEN deletions in the non-amplified carcinomas $(P<0.001)$. Moreover, PTEN deletion was very commonly found $(P<0.001)$ in tumours with HER2 overexpression (13 out of $18,72.2 \%$ ), while rarely detected in HER2-negative tumours (32 out of 177, 18.1\%). Considering all tumours indicative for anti-HER2 therapy (overexpression and amplification), 14 out of 23 (60.8\%) showed a genomic loss of PTEN $(P<0.001)$. Homozygous deletion of PTEN was stronger associated with EGFR CNG or HER2 amplification (10 out of $16,62.5 \%$ and 6 out of $16,37.5 \%$, respectively) than hemizygous deletion ( 8 out of $29,27.6 \%$ and 1 out of $28,3.6 \%$, respectively, Table 1$)$

Deletion of genomic PTEN was strongly associated with immunohistochemical loss of PTEN expression $(P<0.001)$. In all, $50.0 \%$ (24 out of 48 ) of the tumours with PTEN deletion $(48.5 \%$ of hemizygous and $53.3 \%$ of homozygous deletion) also indicated loss of PTEN protein expression in contrast to $14.1 \%$ (24 out of 170) PTEN expression loss in non-deleted cases. Hemi- and homozygous deletion of PTEN were associated with lower diseasespecific survival rates in univariate Kaplan-Meier analysis revealing even worse prognosis for homozygous deletion (Figure 2). Prognostic significance of genomic PTEN was independent of grading, EGFR and HER2 status. Deletion of PTEN (hemi- and homozygous) harboured an unfavourable outcome in both EGFR-negative $(P<0.001)$ and -positive cases $(P=0.039)$. Similarly, loss of PTEN indicated worse prognosis in HER2negative $(P=0.003)$ and HER2-positive (amplification and overexpression, $P=0.027$ ) tumours. With regard to grading, PTEN deletion went along with lower survival rates in both low/intermediate grade $(P=0.002)$ and high-grade tumours 

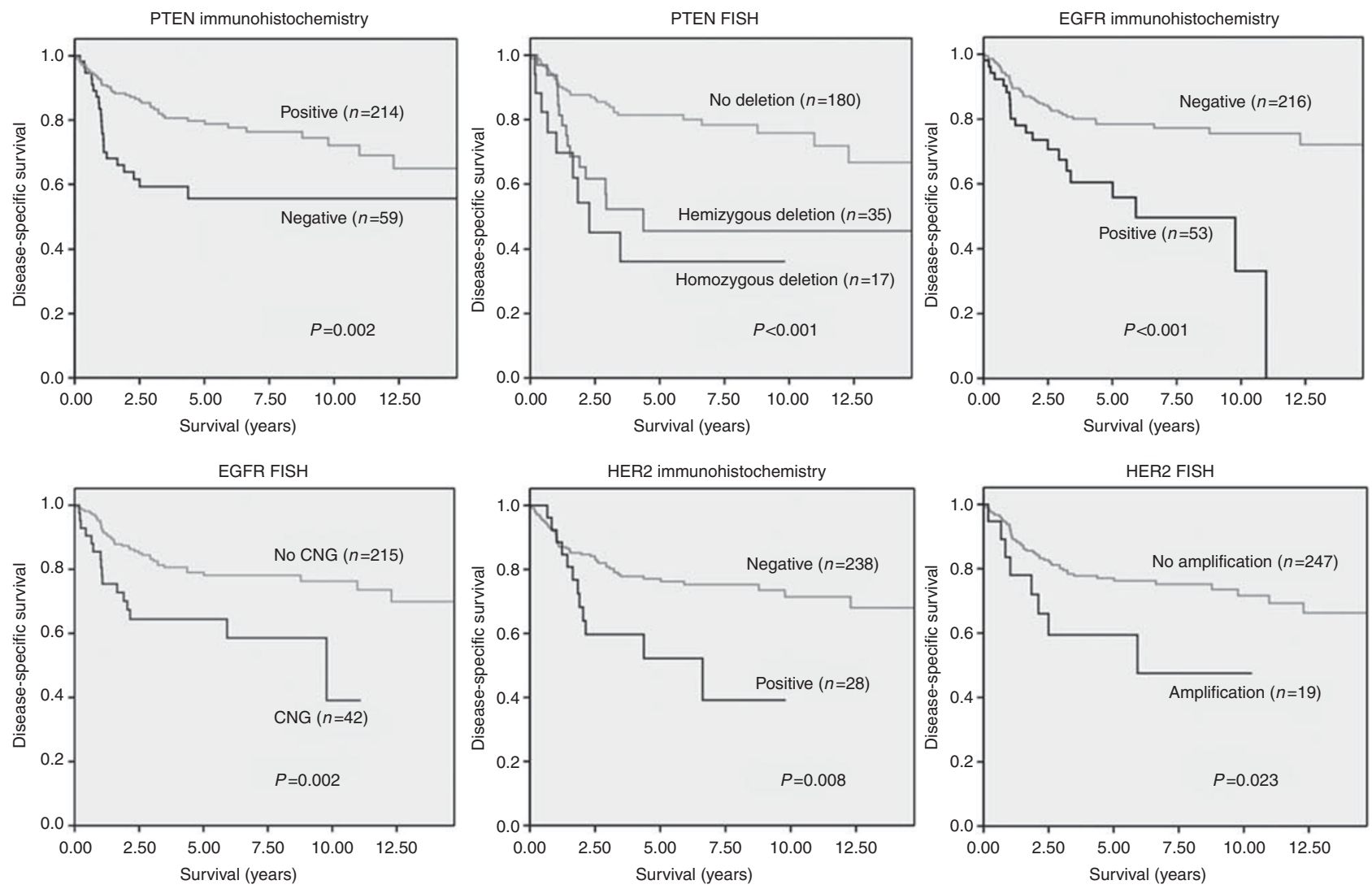

Figure 2 Univariate Kaplan-Meier survival analyses for IHC and FISH of PTEN, EGFR and HER2.

$(P=0.120)$, although not reaching statistical significance in the latter category. Moreover, deletion of PTEN (hemi- and homozygous) evolved as a strong negative predictor from multivariate Cox regression analysis (Table 2 ).

\section{PTEN IHC}

Results of combined nuclear and cytoplasmic PTEN staining $(n=273)$ are shown in Table 1 . The average PTEN IRS was 122 (range $0-300)$. Negative PTEN expression (IRS $<60$ ) was identified in $21.6 \%(n=59)$ of all tumours and was most frequently found in SDCs (41.7\%), SQCCs (37.0\%) and ACNOS (29.6\%). Loss of PTEN expression was associated with advanced age $(P=0.005)$, highgrade malignancy $(P<0.001)$, increased tumour size $(P=0.036)$, lymph node metastases $(P=0.007)$ and advanced (III, IV) tumour stage $(P=0.004)$.

Loss of PTEN expression was also correlated to an increased gene copy number of EGFR, because $45.5 \%$ (15 out of 33) of the tumours with a CNG of EGFR presented a negative PTEN expression in comparison with $16.4 \%$ (30 out of 83 ) PTEN negativity in tumours without a CNG of EGFR $(P=0.001)$. Negative PTEN expression more frequently occurred in carcinomas overexpressing EGFR protein (14 out of 49,28.6\%) than in those without EGFR overexpression (35 out of 188, 18.6\%), although not reaching statistical significance $(P=0.164)$. In all, 10 out of $19(52.6 \%)$ carcinomas with an overexpression of HER2 protein showed an immunohistochemical loss of PTEN in comparison with $17.5 \%$ (38 out of 217) PTEN negativity in HER2-negative tumours $(P<0.001)$. Phosphatase and tensin homologue located on chromosome 10 was more often absent in HER2-amplified tumours (4 out of 11,36.4\%) than in those without HER2 amplification (43 out of $214,20.1 \%, P=0.247$ ). In univariate analysis, loss of PTEN expression was significantly
Table 2 Univariate (Kaplan-Meier - log-rank) and multivariate (Cox regression - Enter) analysis

\begin{tabular}{llrccc}
\hline & & & \multicolumn{2}{c}{ Multivariate } \\
\cline { 4 - 6 } Variable & Coding & Univariate & Log-rank & Significance & HR $(\mathbf{9 5} \% \mathbf{C l})$ \\
\hline Grade & GI/G2 vs G3 & $<\mathbf{0 . 0 0 1}$ & 0.052 & $2.56(0.99-6.60)$ \\
T-stage & I, 2 vs 3,4 & $<\mathbf{0 . 0 0 1}$ & $\mathbf{0 . 0 2 9}$ & $2.50(1.10-5.69)$ \\
N-stage & O vs I, 2, 3 & $<\mathbf{0 . 0 0 1}$ & 0.061 & $2.06(0.97-4.38)$ \\
R-stage & RO vs RI/R2 & $<\mathbf{0 . 0 0 1}$ & $\mathbf{0 . 0 0 4}$ & $3.03(1.43-6.43)$ \\
EGFR FISH & No CNG vs CNG & $\mathbf{0 . 0 0 2}$ & 0.964 & $1.02(0.40-2.64)$ \\
EGFR IHC & Neg vs Pos & $<\mathbf{0 . 0 0 1}$ & 0.111 & $1.94(0.86-4.39)$ \\
HER2 FISH & No Ampl vs Ampl. & $\mathbf{0 . 0 2 3}$ & 0.634 & $0.71(0.18-2.89)$ \\
HER2 IHC & Neg vs Pos & $\mathbf{0 . 0 0 8}$ & 0.301 & $0.60(0.23-1.58)$ \\
PTEN FISH & No Del vs Del & $<\mathbf{0 . 0 0 1}$ & $\mathbf{0 . 0 1 2}$ & $3.17(1.29-7.78)$ \\
PTEN IHC & Pos vs Neg & $\mathbf{0 . 0 0 2}$ & 0.397 & $1.49(0.59-3.77)$ \\
\hline
\end{tabular}

Abbreviations: $\quad$ Ampl = amplification; $\quad \mathrm{Cl}=$ confidence interval; $\quad \mathrm{CNG}=$ copy number gain; Del $=$ deletion; $E G F R=$ epidermal growth factor receptor; $\mathrm{FISH}=$ fluorescence in situ hybridisation; HER2 = human epidermal growth factor receptor 2; $\mathrm{HR}=$ hazard ratio; $\mathrm{IHC}=$ immunohistochemistry; $\mathrm{n}=$ number of patients; Neg = negative; Pos = positive; PTEN = phosphatase and tensin homologue located on chromosome 10; $\mathrm{R}=$ residual tumour. Statistically significant associations are highlighted in bold.

associated with unfavourable long-term survival $(P=0.002$, Figure 2).

\section{EGFR and HER2}

Fluorescence in situ hybridisation analysis for EGFR was available for 257 cases. In all, $42(16.3 \%)$ tumour specimens presented an increased gene copy number (39 high polysomies and 
3 amplifications). Epidermal growth factor receptor positivity was associated with high-grade carcinomas $(P<0.001)$, tumour-size $(P=0.003)$, lymph node metastases $(P=0.003)$ and advanced (III, IV) tumour stage $(P<0.001)$. In univariate Kaplan-Meier analysis CNG of EGFR predicted worse survival $(P=0.002$, Figure 2). Immunohistochemical analysis of EGFR was available for 269 cases. In total, 53 tumours (19.7\%) presented overexpression of EGFR. Epidermal growth factor receptor positivity was associated with age $>70(P<0.001)$, high-grade malignancy $(P=0.001)$ and worse overall survival in univariate analysis $(P<0.001$, Figure 2$)$. Increased EGFR copy number was strongly correlated to EGFR overexpression $(P<0.001)$.

Human epidermal growth factor receptor 2 FISH analysis was performed for 266 cases. In 19 cases (7.1\%), amplification (11 SDCs) occurred and was associated with positive lymph nodes $(P=0.008)$, high-grade malignancy $(P<0.001)$ and unfavourable overall survival $(P=0.023$, Figure 2$)$.

In all, 28 out of $266(10.5 \%)$ investigated cases (16 SDCs) presented immunohistochemical overexpression of HER2. Human epidermal growth factor receptor 2 positivity was associated with positive lymph nodes $(P<0.001)$, advanced tumour stage $(P=0.004)$, high-grade malignancy $(P<0.001)$ and worse survival $(P=0.008$, Figure 2). Human epidermal growth factor receptor 2 amplification was strongly associated with HER2 overexpression $(P<0.001)$. Moreover, HER2 amplification was associated with CNG of EGFR $(P=0.013)$.

\section{DISCUSSION}

This study investigated the role of the tumour-suppressor PTEN in a considerable and representative number of salivary gland cancer. By use of interphase FISH analysis, it revealed for the first time a hemi- or homozygous deletion of the gene on chromosome $10 \mathrm{q} 23.3$ in $>20 \%$ of these tumours, especially in SDCs, ACNOS and SQCCs. Homozygous deletion of PTEN (7.3\% of all tumours) was almost exclusively (94.1\%) found in high-grade malignancies pointing towards a more aggressive growth pattern in comparison with hemizygous gene deletion. This observation is in accordance with the hypothesis that hemizygous loss of PTEN with haploinsufficiency of the remaining allele leads to genomic instability and cancer development as shown by in vivo studies on knockout PTEN mice (Di Cristofano et al, 1998; Kwabi-Addo et al, 2001; Shen-Li et al, 2011), while complete inactivation accelerates tumour dedifferentiation, progression and metastases (Verhagen et al, 2006; Schmitz et al, 2007). In our study, both hemi- and homozygous genomic deletion of PTEN strongly correlated with immunohistochemical loss of the protein expression. Loss of PTEN function, especially deletion of genomic PTEN independently indicated an unfavourable clinical course of patients with lymph node metastases, rapid tumour progression and worse overall survival. In line with the above mentioned hypothesis, homozygous deletion displayed an even worse prognosis compared with hemizygous deletion in the investigated salivary gland carcinomas. Downregulation of PTEN with negative impact on prognosis has already been described for prostate cancer, colorectal and breast cancer and high-grade gliomas (Korshunov et al, 2004; Fujita et al, 2006; Frattini et al, 2007; Yoshimoto et al, 2007; Laurent-Puig et al, 2009).

Different types of salivary gland carcinomas show overexpression and an increased gene copy number of EGFR and HER2 with negative impact on prognosis (Press et al, 1994; Ettl et al, 2008; Lujan et al, 2010; Williams et al, 2010). In this study, $16.3 \%$ of the carcinomas presented high polysomy or amplification of the EGFR gene and $19.7 \%$ showed overexpression of the EGFR protein. Overexpression and amplification of HER2 occurred in 10.5\% and $7.1 \%$ of cases, respectively, especially in SDCs. Both EGFR and HER2 were associated with negative clinicopathological parameters and unfavourable survival. Increased gene copy number of EGFR and overexpression/amplification of HER2 pose the precondition for anti-EGFR and anti-Her2 therapy in breast, colorectal and lung cancer (Wolff et al, 2007; Hirsch et al, 2008; Laurent-Puig et al, 2009). Moreover, in salivary gland cancer first phase-II trials on targeted therapies have been conducted and revealed that the use of anti-EGFR agents, such as cetuximab and gefitinib, failed to produce obvious response, although the majority of patients showed disease stabilisation (Glisson et al, 2005; Locati et al, 2009). These studies mainly comprised adenoid cystic carcinomas $(68-77 \%)$ and the EGFR status was characterised by IHC. In the investigation of Locati et al (2009), tumours were also retrospectively analysed by FISH, however, no amplification or high polysomy of EGFR was found in these tumours. This might have been a reason for missing response, as EGFR protein expression observed by IHC has been described as an unreliable predictor of responsiveness to EGFR inhibitors (Ciardiello and Tortora, 2008; Vidal et al, 2009). The use of trastuzumab (HER2inhibitor) and lapatinib (combined TKI against EGFR and HER2) did also show no obvious reactivity in ACCs (Haddad et al, 2003; Agulnik et al, 2007; Vidal et al, 2009). However, partial response to trastuzumab was reported for a mucoepidermoid carcinoma with HER2 3 + immunostaining (Haddad et al, 2003) and prolonged tumour progression to lapatinib was documented in three nonACC tumours with HER2 amplification and $3+$ staining for EGFR and HER2 (Vidal et al, 2009). Therefore, increased gene copy number of EGFR and amplification or overexpression of HER2 should be a precondition for an anti-EGFR and anti-HER2 therapy in salivary gland cancer, too.

In this study, we demonstrate that deletion of genomic PTEN, in particular homogenous deletion, predominantly occurs in salivary gland carcinomas with an increased copy number of EGFR or amplification of HER2. Moreover, loss of PTEN protein expression is frequently found in tumours with EGFR and HER2 overexpression. This coincidence of PTEN loss and EGFR/HER2 gene/expression gain most likely represents an accumulation of independent genetic disorders in poorly differentiated carcinomas during tumourigenesis rather than a functional relationship between EGFR, HER2 and PTEN. Owing to its function as a tumour suppressor, however, there is some evidence that PTEN has significant impact on the efficiency of targeted therapies by antagonising the activation of the PI $3 \mathrm{~K}-\mathrm{AKT}$ pathway that is predominantly activated by EGF-/HER2-receptor tyrosine kinases in different tumour types. In successfully treated cancers, PI3K signalling is attenuated or even turned off by EGFR- or HER2targeting (using antibodies or receptor kinase inhibitors). If there is a loss of PTEN expression/function, however, PI3K signalling remains active in spite of anti-EGFR or anti-HER2 therapy (Courtney et al, 2010; Nahta and O'Regan, 2010). In breast cancer, (active), PTEN has been reported to be an essential parameter for responsiveness to trastuzumab treatment, while loss of PTEN rather predicts resistance to trastuzumab in HER2-positive patients (Nagata et al, 2004; Pandolfi, 2004; Fujita et al, 2006). On the other hand, the additional use of a PI3K inhibitor is reported to overcome the PTEN loss-induced trastuzumab resistance (Courtney et al, 2010). In colorectal cancer, high polysomy or amplification of EGFR was associated with response to cetuximab therapy, whereas loss of PTEN expression, (evaluated by IHC) rather is indicative for therapy resistance (Frattini et al, 2007; Laurent-Puig et al, 2009; Loupakis et al, 2009). The same observation was made in glioblastomas, where expression of PTEN was associated with clinical response to the EGFR kinase inhibitors gefitinib and erlotinib (Mellinghoff et al, 2005). For these tumours, evidence derived from in vitro analysis suggests that resistance can be overcome by coupling anti-EGFR-agents with mTOR inhibitors (Wang et al, 2006).

In anti-EGFR/Her2-treated salivary gland cancer patients, a retrospective analysis could elucidate the suggested correlation of 
the PTEN status with therapy response rates. As a consequence, PTEN analysis might find the way into routine diagnostics and might facilitate therapy decisions related to anti-EGFR or anti-HER2 targeting. Additional targeting of the PI3K pathway potentially enhances therapy efficiency in salivary gland cancer.

Despite the significant findings in this study it has to be kept in mind that the investigated salivary gland carcinomas we investigated comprise a variety of subtypes with inter- and intratumoural differences and characteristics. This fact aggravates further preclinical and clinical investigations of these rare tumours.

In conclusion, this is the first report on a reduced PTEN function in different types of salivary gland cancer indicating worse prognosis. We could demonstrate an association between

\section{REFERENCES}

Agulnik M, Cohen EW, Cohen RB, Chen EX, Vokes EE, Hotte SJ, Winquist E, Laurie S, Hayes DN, Dancey JE, Brown S, Pond GR, Lorimer I, Daneshmand M, Ho J, Tsao MS, Siu LL (2007) Phase II study of lapatinib in recurrent or metastatic epidermal growth factor receptor and/or erbB2 expressing adenoid cystic carcinoma and non adenoid cystic carcinoma malignant tumors of the salivary glands. J Clin Oncol 25: $3978-3984$

Barnes L, Eveson JW, Rei, DS (2005) Pathology and Genetics of Head and Neck Tumours. World Health Organization Classification of Tumours Pathology and genetics of head and neck tumours. World Health Organization Classification of Tumours. IARC Press: Lyon

Carracedo A, Alimonti A, Pandolfi PP (2011) PTEN level in tumor suppression: how much is too little? Cancer Res 71: 629-633

Chung CH, Ely K, McGavran L, Varella-Garcia M, Parker J, Parker N, Jarrett C, Carter J, Murphy BA, Netterville J, Burkey BB, Sinard R, Cmelak A, Levy S, Yarbrough WG, Slebos RJ, Hirsch FR (2006) Increased epidermal growth factor receptor gene copy number is associated with poor prognosis in head and neck squamous cell carcinomas. J Clin Oncol 24: $4170-4176$

Ciardiello F, Tortora G (2008) EGFR antagonists in cancer treatment. $N$ Engl J Med 358: $1160-1174$

Courtney KD, Corcoran RB, Engelman JA (2010) The PI3K pathway as drug target in human cancer. J Clin Oncol 28: 1075-1083

Di Cristofano A, Pesce B, Cordon-Cardo C, Pandolfi PP (1998) PTEN is essential for embryonic development and tumour suppression. Nat Genet 19: $348-355$

Elston CW, Ellis IO (1991) Pathological prognostic factors in breast cancer. I. The value of histological grade in breast cancer: experience from a large study with long-term follow-up. Histopathology 19: $403-410$

Ettl T, Schwarz S, Kleinsasser N, Hartmann A, Reichert TE, Driemel O (2008) Overexpression of EGFR and absence of C-KIT expression correlate with poor prognosis in salivary gland carcinomas. Histopathology 53: $567-577$

Eveson JW, Auclair P, Gnepp DR, El-Naggar AK (2005) Tumours of the salivary glands: introduction. In Pathology and Genetics of Head and Neck Tumours. World Health Organization Classification of Tumours, Barnes L, Eveson JW, Reichart P, Sidransky D (eds), pp. 212-215. IARC Press: Lyon

Frattini M, Saletti P, Romagnani E, Martin V, Molinari F, Ghisletta M, Camponovo A, Etienne LL, Cavalli F, Mazzucchelli L (2007) PTEN loss of expression predicts cetuximab efficacy in metastatic colorectal cancer patients. Br J Cancer 97: 1139-1145

Fujita T, Doihara H, Kawasaki K, Takabatake D, Takahashi H, Washio K, Tsukuda K, Ogasawara Y, Shimizu N (2006) PTEN activity could be a predictive marker of trastuzumab efficacy in the treatment of ErbB2overexpressing breast cancer. Br J Cancer 94: 247-252

Glisson BS, Blumenschein G, Francisco M, Erasmus J, Zinner R, Kies MS (2005) Phase II trial on gefitinib in patients with incurable salivary gland cancer [abstract]. J Clin Oncol 23: 5532

Haddad R, Colevas AD, Krane JF, Cooper D, Glisson B, Amrein PC, Weeks L, Costello R, Posner M (2003) Herceptin in patients with advanced or metastatic salivary gland carcinomas. A phase II study. Oral Oncol 39: $724-727$
PTEN loss and EGFR and HER2 signalling, which might influence response to targeted therapies.

\section{ACKNOWLEDGEMENTS}

We wish to thank Rudolf Jung for his excellent immunohistochemical stainings, Anja Reck for assistance in acquiring follow-up data and Mrs Marietta Bock for her excellent technical assistance in FISH analysis.

\section{Conflict of interest}

The authors declare no conflict of interest.
Hirsch FR, Herbst RS, Olsen C, Chansky K, Crowley J, Kelly K, Franklin WA, Bunn Jr PA, Varella-Garcia M, Gandara DR (2008) Increased EGFR gene copy number detected by fluorescent in situ hybridization predicts outcome in non-small-cell lung cancer patients treated with cetuximab and chemotherapy. I Clin Oncol 26: 3351-3357

Jouzdani E, Yachouh J, Costes V, Faillie JL, Cartier C, Poizat F, Pierre G, Burcia V, Makeieff M, Crampette L, Guerrier B, Garrel R (2010) Prognostic value of a three-grade classification in primary epithelial parotid carcinoma: result of a histological review from a 20-year experience of total parotidectomy with neck dissection in a single institution. Eur J Cancer 46: 323-331

Korshunov A, Sycheva R, Golanov A (2004) Molecular stratification of diagnostically challenging high-grade gliomas composed of small cells: the utility of fluorescence in situ hybridization. Clin Cancer Res 10: $7820-7826$

Kwabi-Addo B, Giri D, Schmidt K, Podsypanina K, Parsons R, Greenberg N, Ittmann M (2001) Haploinsufficiency of the PTEN tumor suppressor gene promotes prostate cancer progression. Proc Natl Acad Sci USA 98: $11563-11568$

Laurent-Puig P, Cayre A, Manceau G, Buc E, Bachet JB, Lecomte T, Rougier P, Lievre A, Landi B, Boige V, Ducreux M, Ychou M, Bibeau F, Bouche O, Reid J, Stone S, Penault-Llorca F (2009) Analysis of PTEN, BRAF, and EGFR status in determining benefit from cetuximab therapy in wild-type KRAS metastatic colon cancer. J Clin Oncol 27: 5924-5930

Li J, Yen C, Liaw D, Podsypanina K, Bose S, Wang SI, Puc J, Miliaresis C, Rodgers L, McCombie R, Bigner SH, Giovanella BC, Ittmann M, Tycko B, Hibshoosh H, Wigler MH, Parsons R (1997) PTEN, a putative protein tyrosine phosphatase gene mutated in human brain, breast, and prostate cancer. Science 275: $1943-1947$

Locati LD, Bossi P, Perrone F, Potepan P, Crippa F, Mariani L, Casieri P, Orsenigo $M$, Losa $M$, Bergamini C, Liberatoscioli C, Quattrone P, Calderone RG, Rinaldi G, Pilotti S, Licitra L (2008) Cetuximab in recurrent and/or metastatic salivary gland carcinomas: a phase II study. Oral Oncol 45: $574-578$

Locati LD, Bossi P, Perrone F, Potepan P, Crippa F, Mariani L, Casieri P, Orsenigo $M$, Losa $M$, Bergamini $C$, Liberatoscioli $C$, Quattrone $P$, Calderone RG, Rinaldi G, Pilotti S, Licitra L (2009) Cetuximab in recurrent and/or metastatic salivary gland carcinomas: a phase II study. Oral Oncol 45: $574-578$

Loupakis F, Pollina L, Stasi I, Ruzzo A, Scartozzi M, Santini D, Masi G, Graziano F, Cremolini C, Rulli E, Canestrari E, Funel N, Schiavon G, Petrini I, Magnani M, Tonini G, Campani D, Floriani I, Cascinu S, Falcone A (2009) PTEN expression and KRAS mutations on primary tumors and metastases in the prediction of benefit from cetuximab plus irinotecan for patients with metastatic colorectal cancer. J Clin Oncol 27: $2622-2629$

Lujan B, Hakim S, Moyano S, Nadal A, Caballero M, Diaz A, Valera A, Carrera M, Cardesa A, Alos L (2010) Activation of the EGFR/ERK pathway in high-grade mucoepidermoid carcinomas of the salivary glands. Br J Cancer 103: 510 - 516

Mellinghoff IK, Wang MY, Vivanco I, Haas-Kogan DA, Zhu S, Dia EQ, Lu KV, Yoshimoto K, Huang JH, Chute DJ, Riggs BL, Horvath S, Liau LM, Cavenee WK, Rao PN, Beroukhim R, Peck TC, Lee JC, Sellers WR, Stokoe D, Prados M, Cloughesy TF, Sawyers CL, Mischel PS (2005) Molecular 
determinants of the response of glioblastomas to EGFR kinase inhibitors. N Engl J Med 353: 2012-2024

Milanes-Yearsley M, Hammond ME, Pajak TF, Cooper JS, Chang C, Griffin T, Nelson D, Laramore G, Pilepich M (2002) Tissue micro-array: a cost and time-effective method for correlative studies by regional and national cancer study groups. Mod Pathol 15: 1366-1373

Nagata Y, Lan KH, Zhou X, Tan M, Esteva FJ, Sahin AA, Klos KS, Li P, Monia BP, Nguyen NT, Hortobagyi GN, Hung MC, Yu D (2004) PTEN activation contributes to tumor inhibition by trastuzumab, and loss of PTEN predicts trastuzumab resistance in patients. Cancer Cell 6: $117-127$

Nahta R, O'Regan RM (2010) Evolving strategies for overcoming resistance to HER2-directed therapy: targeting the PI3K/Akt/mTOR pathway. Clin Breast Cancer 10(Suppl 3): S72-S78

Negri FV, Bozzetti C, Lagrasta CA, Crafa P, Bonasoni MP, Camisa R, Pedrazzi G, Ardizzoni A (2010) PTEN status in advanced colorectal cancer treated with cetuximab. Br J Cancer 102: $162-164$

Pandolfi PP (2004) Breast cancer-loss of PTEN predicts resistance to treatment. $N$ Engl J Med 351: 2337-2338

Pectasides E, Rampias T, Kountourakis P, Sasaki CT, Kowalski D, Fountzilas G, Zaramboukas T, Rimm DL, Burtness B, Psyrri A (2011) Comparative prognostic value of EGFR protein expression compared with FISH for head and neck squamous cell carcinoma (HNSCC). Clin Cancer Res 17: 2947-2954

Press MF, Pike MC, Hung G, Zhou JY, Ma Y, George J, Dietz-Band J, James W, Slamon DJ, Batsakis JG et al. (1994) Amplification and overexpression of HER-2/neu in carcinomas of the salivary gland: correlation with poor prognosis. Cancer Res 54: 5675-5682

Sassen A, Rochon J, Wild P, Hartmann A, Hofstaedter F, Schwarz S, Brockhoff G (2008) Cytogenetic analysis of HER1/EGFR, HER2, HER3 and HER4 in 278 breast cancer patients. Breast Cancer Res 10: R2

Schmitz M, Grignard G, Margue C, Dippel W, Capesius C, Mossong J, Nathan M, Giacchi S, Scheiden R, Kieffer N (2007) Complete loss of PTEN expression as a possible early prognostic marker for prostate cancer metastasis. Int J Cancer 120: 1284-1292

Schwarz S, Stiegler C, Müller M, Ettl T, Brockhoff G, Zenk J, Agaimy A (2011) Salivary gland mucoepidermoid carcinoma is a clinically, morphologically and genetically heterogeneous entity: a clinicopathological study of 40 cases with emphasis on grading, histological variants and presence of the $\mathrm{t}(11 ; 19)$ translocation. Histopathology 58: $557-570$
Shen-Li H, Koujak S, Szablocs M, Parsons R (2011) Reduction of PTEN dose leads to neoplastic development in multiple organs of PTEN (shRNA) mice. Cancer Biol Ther 10: 1194-1200

Sircar K, Yoshimoto M, Monzon FA, Koumakpayi IH, Katz RL, Khanna A, Alvarez K, Chen G, Darnel AD, Aprikian AG, Saad F, Bismar TA, Squire JA (2009) PTEN genomic deletion is associated with p-Akt and AR signalling in poorer outcome, hormone refractory prostate cancer. J Pathol 218: $505-513$

Sobin LH, Gospodarowicz MK, Wittekind C (2009) TNM Classification of Malignant Tumours (UICC). Wiley-Liss, Inc.: Chichester, West Sussex, UK

Therkildsen MH, Christensen M, Andersen LJ, Schiodt T, Hansen HS (1998) Salivary gland carcinomas-prognostic factors. Acta Oncol 37: $701-713$

Verhagen PC, van Duijn PW, Hermans KG, Looijenga LH, van Gurp RJ, Stoop H, van der Kwast TH, Trapman J (2006) The PTEN gene in locally progressive prostate cancer is preferentially inactivated by bi-allelic gene deletion. J Pathol 208: 699-707

Vidal L, Tsao MS, Pond GR, Cohen EE, Cohen RB, Chen EX, Agulnik M, Hotte S, Winquist E, Laurie S, Hayes DN, Ho J, Dancey J, Siu LL (2009) Fluorescence in situ hybridization gene amplification analysis of EGFR and HER2 in patients with malignant salivary gland tumors treated with lapatinib. Head Neck 31: 1006-1012

Wang MY, Lu KV, Zhu S, Dia EQ, Vivanco I, Shackleford GM, Cavenee WK, Mellinghoff IK, Cloughesy TF, Sawyers CL, Mischel PS (2006) Mammalian target of rapamycin inhibition promotes response to epidermal growth factor receptor kinase inhibitors in PTEN-deficient and PTEN-intact glioblastoma cells. Cancer Res 66: 7864-7869

Williams MD, Roberts DB, Kies MS, Mao L, Weber RS, El-Naggar AK (2010) Genetic and expression analysis of HER-2 and EGFR genes in salivary duct carcinoma: empirical and therapeutic significance. Clin Cancer Res 16: 2266-2274

Wolff AC, Hammond ME, Schwartz JN, Hagerty KL, Allred DC, Cote RJ, Dowsett M, Fitzgibbons PL, Hanna WM, Langer A, McShane LM, Paik S, Pegram MD, Perez EA, Press MF, Rhodes A, Sturgeon C, Taube SE, Tubbs R, Vance GH, van de Vijver M, Wheeler TM, Hayes DF (2007) American Society of Clinical Oncology/College of American Pathologists guideline recommendations for human epidermal growth factor receptor 2 testing in breast cancer. J Clin Oncol 25: 118-145

Yoshimoto M, Cunha IW, Coudry RA, Fonseca FP, Torres CH, Soares FA, Squire JA (2007) FISH analysis of 107 prostate cancers shows that PTEN genomic deletion is associated with poor clinical outcome. Br J Cancer 97: $678-685$

This work is published under the standard license to publish agreement. After 12 months the work will become freely available and the license terms will switch to a Creative Commons Attribution-NonCommercial-Share Alike 3.0 Unported License. 